PROCEEDINGS OF THE

AMERICAN MATHEMATICAL SOCIETY

Volume 131, Number 11, Pages 3365-3369

S 0002-9939(03)07155-7

Article electronically published on June 19, 2003

\title{
THE DERKSEN INVARIANT VS. THE MAKAR-LIMANOV INVARIANT
}

\author{
ANTHONY CRACHIOLA AND STEFAN MAUBACH
}

(Communicated by Bernd Ulrich)

\begin{abstract}
In this article it is shown that the Makar-Limanov invariant of a ring (or variety) can be trivial while the Derksen invariant is not, and vice versa.
\end{abstract}

\section{INTRODUCTION}

The Makar-Limanov invariant was introduced by Makar-Limanov in [5] to prove that the variety in $\mathbb{C}^{4}$ given by the equation $X^{2} Y+X+Z^{2}+T^{3}$ is not isomorphic to $\mathbb{C}^{3}$. Later on, Derksen gave an alternative proof in [3] by introducing a different invariant. The general idea was that both invariants are kind of dual, in the sense that they can distinguish the same set of rings from the polynomial rings. However, this article gives examples in which this is not the case, thus stating that the invariants are clearly different.

\section{Definitions and notations}

In this section, $R$ denotes a commutative finitely generated $\mathbb{C}$-algebra and $\mathbb{N}$ the non-negative integers.

Definition 2.1. $\quad$ (i) A map $D: R \longrightarrow R$ is called a derivation if it satisfies the Leibniz rule: $D(a b)=a D(b)+D(a) b$ for all $a, b \in R$.

(ii) A derivation is called locally nilpotent if for each $a \in R$ there exists $n \in \mathbb{N}$ such that $D^{n}(a)=0$.

(iii) When $D$ is a locally nilpotent derivation, we denote by $R^{D}$ the kernel of the map $D$, i.e. $R^{D}:=\{a \in R \mid D(a)=0\}$.

(iv) $L N D(R)$ is the set of all locally nilpotent derivations on $R$.

(v) $L N D^{*}(R):=L N D(R) \backslash\{0\}$ (notice the zero map "0" is actually a derivation).

(vi) $M L(R):=\bigcap_{D \in L N D(R)} R^{D}$, the Makar-Limanov invariant of $R \rrbracket$

(vii) $H D(R)$ is the $\mathbb{C}$-algebra generated by $\bigcup_{D \in L N D^{*}(R)} R^{D}$ 目

Received by the editors June 12, 2002.

2000 Mathematics Subject Classification. Primary 14R05; Secondary 13N15.

Key words and phrases. Makar-Limanov invariant, Derksen invariant, ring invariant, locally nilpotent derivation.

${ }^{1}$ The original notation introduced by Makar-Limanov himself was $A K(R)$, "absolute kernel", and this notation is sometimes used too.

${ }^{2}$ This invariant is often denoted by " $D(R)$ " but since $D$ is a very common notation for a derivation, the notation " $H D$ " (for Harm Derksen) came into fashion. 
Example 2.2. If $R=\mathbb{C}\left[X_{1}, \ldots, X_{n}\right]$, then $M L(R)=\mathbb{C}$, and in the case $n \geq 2$, $H D(R)=R$. In the case $n=1, H D(R)=\mathbb{C}$ (a small exception).

Corollary 2.3. If $M L(R) \neq \mathbb{C}$ (i.e. $M L(R)$ is larger than $\mathbb{C}$ ), then $R$ is not a polynomial ring. If $\operatorname{dim}(R) \geq 2$ and $H D(R) \neq R$, then $R$ is not a polynomial ring.

\section{SPECIFIC RINGS AND THEIR INVARIANTS}

3.1. Makar-Limanov invariant trivial, Derksen invariant not. In this section we will give a ring whose Makar-Limanov invariant is trivial but its Derksen invariant is not.

Definition 3.1. Define the ideal $I:=(X, Y) \subset \mathbb{C}[X, Y]$, and let

$$
\begin{aligned}
R & :=\mathbb{C}\left[X^{2}, X^{3}, Y^{3}, Y^{4}, Y^{5}, X^{1+i} Y^{1+j} \mid i, j \in \mathbb{N}\right] \\
& =\mathbb{C}\left[X^{2}, X^{3}, Y^{3}, Y^{4}, Y^{5}, X Y, X^{2} Y, X Y^{2}, X Y^{3}\right]
\end{aligned}
$$

(i.e. $\left.R=\mathbb{C} \oplus \mathbb{C} X^{2} \oplus \mathbb{C} X Y \oplus I^{3}\right)$.

Notice that $R$ is finitely generated, Noetherian, and a domain.

Lemma 3.2. $M L(R)=\mathbb{C}$.

Proof. Let $D_{1}:=Y^{3} \partial_{X}$ and $D_{2}:=X^{2} \partial_{Y}$. These are locally nilpotent derivations on $R$, as can be easily checked. Then $R^{D_{1}}=R \cap \mathbb{C}[X, Y]^{D_{1}} \subseteq \mathbb{C}[X, Y]^{D_{1}}=\mathbb{C}[Y]$. Also $R^{D_{2}}=R \cap \mathbb{C}[X, Y]^{D_{2}} \subseteq \mathbb{C}[X, Y]^{D_{2}}=\mathbb{C}[X]$. Thus $\mathbb{C} \subseteq M L(\mathbb{C}) \subseteq R^{D_{1}} \cap R^{D_{2}} \subseteq$ $\mathbb{C}[X, Y]^{D_{1}} \cap \mathbb{C}[X, Y]^{D_{2}}=\mathbb{C}[Y] \cap \mathbb{C}[X]=\mathbb{C}$.

In order to calculate $H D(R)$ we first show that every locally nilpotent derivation on $R$ actually comes from a locally nilpotent derivation on $\mathbb{C}[X, Y]$.

Lemma 3.3. $\quad$ (i) The integral closure of $R$ in $\mathbb{C}[X, Y]$ is $\mathbb{C}[X, Y]$.

(ii) The integral closure of $R$ in $Q(R)$ (the fraction field of $R$ ) is $\mathbb{C}[X, Y]$.

Proof. (i) is easy, since the integral closure of the smaller ring $\mathbb{C}\left[X^{2}, Y^{3}\right]$ in $\mathbb{C}[X, Y]$ is already $\mathbb{C}[X, Y]$.

(ii) $Q(R)=\mathbb{C}(X, Y)$. Let $a \in Q(R)$ be integral over $R$. Then surely $a$ is integral over $\mathbb{C}[X, Y]$. But $\mathbb{C}[X, Y]$ is a UFD and thus integrally closed in its fraction field, i.e., $a \in \mathbb{C}[X, Y]$ already. Thus the integral closure of $R$ in $Q(R)$ is a subset of $\mathbb{C}[X, Y]$. Finally, since $Q(R)=\mathbb{C}(X, Y)$, we are done by part (i).

Notice that if $D$ is a derivation (not necessarily locally nilpotent) on a domain $A$, then it extends uniquely to a derivation on the fraction field $Q(A)$ of $A$, by just forcing $D\left(a^{-1} b\right)=a^{-2}(a D(b)-D(a) b)$ for all $a \in A, b \in A \backslash\{0\}$.

Theorem 3.4 (Seidenberg). Let $A$ be a Noetherian domain containing $\mathbb{Q}, K$ its quotient field and $\tilde{A}$ the integral closure of $A$ in $K$. Let $D$ be a derivation on $A$ and $\tilde{D}$ its unique extension to $K$. Then $\tilde{D}(\tilde{A}) \subseteq \tilde{A}$.

This is quoted literally from [4], prop. 1.2 .15 , page 17 , but it is originally from 10.

Theorem 3.5 (Vasconcelos). Let $A \subseteq B$ be an integral extension where $B$ is a domain and $\mathbb{Q} \subset A$. If $D$ is a derivation on $B$ such that $D A \subseteq A$ and the restriction $\left.D\right|_{A}$ of $D$ to $A$ is locally nilpotent, then $D$ is locally nilpotent.

This is quoted literally from [4], prop. 1.3 .37 , page 29 , but it is originally from [11]. 
Lemma 3.6. If $D$ is a locally nilpotent derivation on $R$, then it extends uniquely to a locally nilpotent derivation on $\mathbb{C}[X, Y] \longrightarrow \mathbb{C}[X, Y]$.

Proof. The integral closure of $R$ in $Q(R)$ is $\mathbb{C}[X, Y]$ (by [3.3). So by the above theorem of Seidenberg $D$ extends uniquely to $\mathbb{C}[X, Y]$. By the above theorem of Vasconcelos we see that $D$ is locally nilpotent.

Lemma 3.7. If $f \in \mathbb{C}[X, Y]$ is a coordinate, $p(T) \in \mathbb{C}[T]$ and $p(f) \in R$, then $X Y$ does not appear as a monomial in $p(f)$.

Proof. $f=f_{0}+f_{1}=f_{0}+a X+b Y+c X^{2}+d X Y+e Y^{2}+g$ for some $g \in I^{3}$ and $a \neq 0$ or $b \neq 0$. Now $p(f)=q\left(f_{1}\right)$ for some $q(T) \in \mathbb{C}[T]$.

$$
\begin{aligned}
q\left(f_{1}\right)= & \lambda_{0}+\lambda_{1} f_{1}+\lambda_{2} f_{1}^{2}+\ldots+\lambda_{n} f_{1}^{n} \\
= & \lambda_{0}+\lambda_{1}\left(a X+b Y+c X^{2}+d X Y+e Y^{2}\right) \\
& \quad+\lambda_{2}\left(a X+b Y+c X^{2}+d X Y+e Y^{2}\right)^{2}+g^{\prime} g^{\prime} \in I^{3}
\end{aligned}
$$

and since $a \neq 0$ or $b \neq 0$ and $q(f) \in R$, we must have $\lambda_{1}=0$. Thus

$$
\begin{aligned}
q(f) & =\lambda_{0}+\lambda_{2}\left(a X+b Y+c X^{2}+d X Y+e Y^{2}\right)^{2}+g^{\prime} g^{\prime} \in I^{3} \\
& =\lambda_{0}+\lambda_{2}\left(a^{2} X^{2}+2 a b X Y+b^{2} Y^{2}\right)+g^{\prime \prime} g^{\prime \prime} \in I^{3},
\end{aligned}
$$

but since the monomial $Y^{2}$ appears in no element of $R$ and $q(f) \in R$, we must have $\lambda_{2} b^{2}=0$ which implies $2 \lambda_{2} a b=0$, which is the coefficient of $X Y$.

Lemma 3.8. Let $D \in L N D(R)$. Suppose there exists $g \in R^{D}$ such that the coefficient of $X Y$ of $g$ is non-zero ( $X Y$ appears in $g$ ). Then $D=0$.

Proof. We know by Lemma 3.6 that $D$ can be extended as a locally nilpotent derivation to $\mathbb{C}[X, Y]$. Suppose $D \neq 0$. Thus $\mathbb{C}[X, Y]^{D}=\mathbb{C}[f]$ for some coordinate $f$ by Rentschler's theorem [9]. Hence $g=p(f) \in R^{D}$. But now by Lemma 3.7, the coefficent of $X Y$ must be zero, a contradiction. Hence our assumption that $D$ was non-zero was wrong, thus $D=0$.

Lemma 3.9. $H D(R) \neq R$.

Proof. If we show that $X Y \notin H D(R)$, then we are done. Suppose $g_{1}, \ldots, g_{n} \in R$ are elements of kernels of non-zero locally nilpotent derivations such that $X Y=$ $p\left(g_{1}, \ldots, g_{n}\right)$ for some $p \in \mathbb{C}\left[T_{1}, \ldots, T_{n}\right]$. Then since $g_{i} \in R$ we have that $g_{i}=c_{i}+$ $a_{i} X^{2}+b_{i} X Y+h_{i}$ for some $a_{i}, b_{i}, c_{i} \in \mathbb{C}, h_{i} \in\left(X^{3}, X^{2} Y, X Y^{2}, Y^{3}\right)$. We may assume that $c_{i}=0$. Furthermore, by Lemma $3.8 b_{i}=0$. Let $p^{\prime}$ be the part of $p$ which is linear. Now $X Y=p^{\prime}\left(a_{1} X^{2}, \ldots, a_{n} X^{2}\right)+h^{\prime}$ for some $h^{\prime} \in\left(X^{3}, X^{2} Y, X Y^{2}, Y^{3}\right)$. This gives a contradiction.

3.2. Derksen invariant trivial, Makar-Limanov invariant not. In this section we will give a class of rings with trivial Derksen invariant but non-trivial Makar-Limanov invariant. Let $A$ be a commutative domain over $\mathbb{C}$ with transcendence degree 1 such that $A$ is not isomorphic to $\mathbb{C}[X]$. For example, take $A$ to be the coordinate ring of a curve which is not isomorphic to the line. We will examine the ring $R:=A\left[X_{1}, \ldots, X_{n}\right]$ for $n \geq 2$.

Lemma 3.10. $H D(R)=R$.

Proof. The kernels of the partial derivatives generate $R$. 
Of course, the same observation shows that a polynomial ring with at least two variables over any algebra always has trivial Derksen invariant. Lemma 3.10 and Example 2.2 are special cases. To show that $M L(R) \neq \mathbb{C}$, we will use the following

Theorem 3.11 (Makar-Limanov). Suppose $A$ is a commutative domain over $\mathbb{C}$ with transcendence degree 1 . Then $M L\left(A\left[X_{1}, \ldots, X_{n}\right]\right)=M L(A)$ for each $n \geq 1$.

This theorem provides an alternate proof of the Abhyankar-Eakin-Heinzer cancellation theorem for curves [1], in the characteristic zero case. For a proof of Theorem 3.11 see [6] or [7. We also need the following standard facts. If $S$ is a commutative domain over $\mathbb{C}$ and $D \in L N D(S)$, then $S^{D}$ is algebraically closed. Moreover, if $D(s)=1$ for some $s \in S$, then $S=S^{D}[s]$. See, for example, [4] for proofs.

Lemma 3.12. $M L(R)=A$.

Proof. By Theorem 3.11 $M L(R)=M L(A)$. Suppose $M L(A) \neq A$, so that there exists a non-zero locally nilpotent derivation $D$ on $A$. Suppose $A^{D} \neq \mathbb{C}$. Then $\operatorname{trdeg}_{\mathbb{C}}\left(A^{D}\right)=1=\operatorname{trdeg}_{\mathbb{C}}(A)$, and so every element of $A$ is algebraically dependent over $A^{D}$. Since $A^{D}$ is algebraically closed, $A^{D}=A$, contradicting the choice of $D$. Hence $A^{D}=\mathbb{C}$. Let $a \in A$ such that $D(a) \in A^{D}$ (always possible with a locally nilpotent derivation), say $D(a)=\lambda$. Let $s=\lambda^{-1} a$, so that $D(s)=1$. Then $A=\mathbb{C}[s]$. This contradicts our original assumption on $A$.

Notice that $\operatorname{trdeg}_{\mathbb{C}}(R)>2$ for the class of rings $R$ in this section. This is a necessary condition for an example of our type. Using the fact that the kernel of a locally nilpotent derivation is algebraically closed, one can show that if $\operatorname{trdeg} \mathbb{C}_{\mathbb{C}}(R)=$ 1 or 2 and $H D(R)=R$, then $M L(R)=\mathbb{C}$.

3.3. Derksen and Makar-Limanov invariants both non-trivial. Finally, in this section we will give a class of rings whose Derksen and Makar-Limanov invariants are both non-trivial and are moreover the same. Let $R$ be the ring over $\mathbb{C}$ given by the equation $x^{n} y=P(z)$, where $n>1$ and $\operatorname{deg}(P)>1$. Danielewski used surfaces of this type to give a negative answer to the generalized Zariski cancellation question [2]. We make use of the following

Theorem 3.13. $L N D(R)=x^{n} \mathbb{C}[x] \frac{\partial}{\partial z}$, where $R$ is viewed as a subring of $\mathbb{C}\left[x, x^{-1}, z\right]$.

For the proof, see [8]. In particular, the kernel of every locally nilpotent derivation on $R$ is $\mathbb{C}[x]$. As a result, we have

Lemma 3.14. $H D(R)=M L(R)=\mathbb{C}[x]$.

\section{ACKNOWLEDGEMENTS}

The first author thanks L. Makar-Limanov for much insight. The second author would like to express his gratitude to A. van den Essen and J. W. Bikker for some stimulating discussions. Both authors would like to thank the organizers of the workshop on group actions on rational varieties (February 27-March 3, 2002) at CRM in Montreal for an inspiring visit. 


\section{REFERENCES}

[1] S. Abhyankar, P. Eakin, and W. Heinzer, On the uniqueness of the coefficient ring in a polynomial ring, J. Algebra, 23(1972), 310-342 MR 46:5300

[2] W. Danielewski, On the cancellation problem and automorphism groups of affine algebraic varieties, preprint, Warsaw, (1989)

[3] H. Derksen, Constructive Invariant Theory and the Linearisation Problem, Ph.D. thesis, University of Basel, (1997)

[4] A. van den Essen, Polynomial Automorphisms and the Jacobian Conjecture, in Progress in Math., Vol. 190, Birkhäuser-Verlag, (2000) MR 2001j:14082

[5] L. Makar-Limanov, On the hypersurface $x+x^{2} y+z^{2}+t^{3}=0$ in $\mathbb{C}^{4}$ or a $\mathbb{C}^{3}$-like threefold which is not $\mathbb{C}^{3}$, Israel J. Math., 96(1996), 419-429 MR 98a:14052

[6] L. Makar-Limanov, Cancellation for curves, preprint

[7] L. Makar-Limanov, Locally nilpotent derivations, a new ring invariant and applications, available at http://www.math.wayne.edu/ $\operatorname{lml}$

[8] L. Makar-Limanov, On the group of automorphisms of a surface $x^{n} y=P(z)$, Israel J. Math., 121(2001), 113-123 MR 2001m:14086

[9] R. Rentschler, Opérations du groupe additif sur le plan affine, C. R. Acad. Sci. Paris, 267(1968), 384-387 MR 38:1093

[10] A. Seidenberg, Derivations and integral closure, Pacific Journal Math., 16(1966), 167-173 MR 32:5686

[11] W. Vasconcelos, Derivations on commutative noetherian rings, Mathematische Zeitschrift, 112(1969), 229-233 MR 40:7247

Department of Mathematics, Wayne State University, Detroit, Michigan 48202

E-mail address: crach@math.wayne.edu

Department of Mathematics, University of Nijmegen, Toernooiveldt, 6525 ED Nijmegen, The Netherlands

E-mail address: stefanm@sci.kun.nl 